\title{
Alprostadil attenuates myocardial ischemia/reperfusion injury by promoting antioxidant activity and eNOS activation in rats ${ }^{1}$
}

\author{
Liping Zhang', Ying Zhang", Xiaofeng Yu'", Huali Xu"v, Dayuan Suiv , Xuezhong Zhao ${ }^{\text {vI }}$
}

'MD, Department of Cardiovascular Medicine, First Hospital, Jilin University, Changchun, Jilin, P.R. China. Acquisition, analysis and interpretation of data; manuscript writing.

"Master, Pharmacology, College of Pharmacy, Jilin University, Changchun, Jilin, P.R. China. Acquisition of data.

I'MD, Pharmacology, College of Pharmacy, Jilin University, Changchun, Jilin, P.R. China. Acquisition of data, technical procedures, histopathological examinations.

IVMD, Pharmacology, College of Pharmacy, Jilin University, Changchun, Jilin, P.R. China. Analysis and interpretation of data.

${ }^{\mathrm{N}} \mathrm{MD}$, Pharmacology, College of Pharmacy, Jilin University, Changchun, Jilin, P.R. China. Conception and design of the study, manuscript writing.

${ }^{V} \mathrm{MD}$, Department of Cardiovascular Medicine, First Hospital, Jilin University, Changchun, Jilin, P.R. China. Conception and design of the study, critical revision, final approval.

\section{Abstract}

Purpose: To investigate the effect of alprostadil on myocardial ischemia/reperfusion $(I / R)$ in rats.

Methods: Rats were subjected to myocardial ischemia for 30 min followed by 24 h reperfusion. Alprostadil (4 or $8 \mu \mathrm{g} / \mathrm{kg}$ ) was intravenously administered at the time of reperfusion and myocardial infarct size, levels of troponin $\mathrm{T}$, and the activity of creatine kinase-MB (CK-MB) and lactate dehydrogenase (LDH) in the serum were measured. Antioxidative parameters, nitric oxide (NO) content and phosphorylated endothelial nitric oxide synthase 3 (p-eNOS) expression in the left ventricles were also measured. Histopathological examinations of the left ventricles were also performed.

Results: Alprostadil treatment significantly reduced myocardial infarct size, serum troponin $T$ levels, and $C K-M B$ and $L D H$ activity $(P<0.05)$. Furthermore, treatment with alprostadil significantly decreased malondialdehyde $(\mathrm{MDA})$ content $(P<0.05)$ and markedly reduced myonecrosis, edema and infiltration of inflammatory cells. Superoxide dismutase and catalase activities $(P<0.05)$, NO level $(P<0.01)$ and p-eNOS $(P<0.05)$ were significantly increased in rats treated with alprostadil compared with control rats.

Conclusion: These results indicate that alprostadil protects against myocardial I/R injury and that these protective effects are achieved, at least in part, via the promotion of antioxidant activity and activation of eNOS.

Key words: Alprostadil. Myocardial ischemia. Nitric Oxide. Rats. 


\section{- Introduction}

The occlusion of coronary arteries interrupts the blood supply to the heart, which causes myocardial ischemia. Men and women aged $40-60$ years are the most susceptible to myocardial ischemia ${ }^{1}$. Myocardial infarction and the associated cell damage and death following ischemia, is the leading cause of adult mortality and morbidity worldwide. Early reperfusion is the only way to salvage ischemic cardiomyocytes, however it still induces reversible and irreversible damage in the cardiomyocytes ${ }^{2}$. Myocardial reperfusion injuries include arrhythmia, transient mechanical dysfunction of the heart, microvascular injury and oxidative stress responses. Oxidative stress caused by the increased generation of reactive oxygen species serves an important role in the pathogenesis of myocardial infarction. Increased oxygen stress damages membrane lipids and proteins, leading to cellular destruction, energy deficiency and cardiomyocyte apoptosis ${ }^{3}$. Nitric oxide (NO) is a fundamental endotheliumderived relaxing factor, which possesses vasodilatory properties and is able to inhibit leukocyte and platelet adherence, as well as suppress smooth muscle cell hyperplasia ${ }^{4}$. NO also limits the severity of myocardial ischemia/reperfusion (I/R) injury ${ }^{5}$. A previous study reported that constitutive endothelial nitrogen oxide synthase 3 (eNOS) expression in the murine ventricular myocardium is required to attenuate myocardial $I / R$ injury and that a deficit of functional eNOS abolishes the cardioprotective effect of early ischemic preconditioning 6 .

Prostaglandin $E_{1} \quad\left(P G E_{1}\right)$ possesses potent direct vasodilator actions and increases local tissue perfusion ${ }^{7}$. A previous study demonstrated that $\mathrm{PGE}_{1}$ has beneficial effects when intravenously administered to patients with chronic vascular disease ${ }^{8}$. Alprostadil is a synthetic form of $P G E_{1}$ that produces a strong relaxant effect on smooth muscles in peripheral blood vessels ${ }^{9}$. Alprostadil interacts with specific G-protein coupled receptors causing an increase in adenosine levels in plasma. Furthermore, alprostadil attenuates morphological alterations and leucocyte adhesion in the renal tissue of rats subjected to renal $I / R^{10}$. It has been demonstrated that alprostadil exerts beneficial therapeutic effects on hepatic I/R injury in rats ${ }^{11}$. In an I/R injury model of the rat sciatic nerve, alprostadil decreased serum malondialdehyde (MDA) content and increased serum NO levels, attenuating peripheral nerve injury ${ }^{12}$. Although it has been determined that alprostadil exhibits protective effects in I/R injuries, to the best of our knowledge, there have been no studies undertaken to evaluate its role in the prevention of myocardial I/R injury. The aim of the present study therefore was to evaluate the effect of alprostadil on myocardial I/R injury in rats.

\section{Methods}

The animals were treated in accordance with the Guide for the Care and Use of Laboratory Animals published by the National Institutes of Health and the present study was approved by the local Ethics Committee of Jilin University (J130926).

Seventy-eight male Sprague-Dawley rats (Age: 8-10 W; Weight: 280-300g) were obtained from the Experimental Animal Center of Jilin University (Changchun, China). Rats were housed in a well-ventilated animal unit under a 12-h light/dark cycle (Temperature: $21 \pm 0.5^{\circ} \mathrm{C}$; Humidity: $\left.40-60 \%\right)$. They had ad libitum access to water and a standard diet.

Experimental groups and study design

Rats were intraperitoneally anesthetized 
with $300 \mathrm{mg} / \mathrm{kg}$ chloral hydrate (Tianjin Bohai Chemical Industry Company, Tianjin, China) and were placed on a heated pad to maintain body temperature at $37^{\circ} \mathrm{C}$. Rat tracheas were cannulated to provide artificial respiration with a ventilator. A thoracotomy was performed in the fourth intercostal space under sterile conditions. A 1.0 silk thread was passed around the left anterior descending coronary artery near its origin using a tapered needle. Both ends of the thread were passed through a $1 \mathrm{~cm}$ long polyethylene tube (outer diameter, $2.0 \mathrm{~mm}$ ), which was used to ligate the coronary artery by pulling the thread. After $30 \mathrm{~min}$ of ischemia, reperfusion was conducted by loosening the thread for $24 \mathrm{~h}$. A total of 18 rats were assigned to the sham group (sham) and were subjected to the same surgery without the left anterior descending coronary artery ligation. A total of 60 rats underwent the occlusion surgery and were randomly divided into three groups $(n=20)$ as follows: A myocardial ischemia/ reperfusion group (I/R), an alprostadil $(4 \mu \mathrm{g} /$ $\mathrm{kg})$ group and an alprostadil $(8 \mu \mathrm{g} / \mathrm{kg}$ ) group. The dosages of 4 and $8 \mu \mathrm{g} / \mathrm{kg}$ alprostadil were selected based on the results of a pilot study in our laboratory (data not published). At the point of reperfusion, rats in the sham and I/R groups were intravenously administered with normal saline. Rats in the alprostadil groups were treated with alprostadil (Harbin Pharmaceutical Group Co., Ltd., Harbin, China) at doses of 4 or $8 \mu \mathrm{g} / \mathrm{kg}$.

\section{Measurement of myocardial infarct size}

At $24 \mathrm{~h}$ after reperfusion, the animals were intraperitoneally administered with 300 $\mathrm{mg} / \mathrm{kg}$ chloral hydrate (Tianjin Bohai Chemical Industry Company, Tianjin, China). Then athoracotomy was performed in the fourth intercostal space and 6 hearts in each group were harvested. The ventricle of heart was cut from the apex to the base into five transverse slices. Slices of rat heart were incubated in 1\% triphenyltetrazolium chloride solution (SigmaAldrich; Merck KGaA, Darmstadt, Germany) at $37^{\circ} \mathrm{C}$ for $15 \mathrm{~min}$ to visualize the infarcted area. Normal myocardium was stained red, whereas the infarcted portion was pale. A laboratory assistant who was blinded to the treatment dissected the ischemic zone from the nonischemic zone. Myocardial infarct size was calculated as: (Weight of ischemic zone/ total weight of the ventricle) $\times 100^{13}$.

Measurement of troponin $T$, creatine kinase$M B(C K-M B)$ and lactate dehydrogenase (LDH)

Following $24 \mathrm{~h}$ reperfusion, the rats were anaesthetized and then blood samples of all animals were harvested and centrifuged at $3,000 \mathrm{~g}$ for $10 \mathrm{~min}$ at $4^{\circ} \mathrm{C}$. The serum was stored at $-80^{\circ} \mathrm{C}$ for biochemical assay. The activities of CK-MB and LDH were evaluated using CK-MB assay kit (No. 20150618) and LDH assay kit (No. 20150810) (Nanching Jiancheng Bioengineering Institute, Nanjing, China) and an automatic biochemical analyzer (Leidu Corporation, Shenzhen, China). Troponin T levels were assayed using an ELISA kit (No. 151112) (Westang Bio-Tech Co., Ltd., Shanghai, China) according to the manufacturer's protocol. All assays were performed in duplicate.

\section{Histopathological examination}

The rats were anaesthetized. The left ventricles of three rats from each group were fixed in $10 \%$ formalin at room temperature for $24 \mathrm{~h}$ and embedded in paraffin. Serial sections ( $5 \mu \mathrm{m}$ thick) were cut and stained with hematoxylin and eosin at room temperature for $2 \mathrm{~min}$. An experienced observer, who was blinded to the treatment, examined the sections using a light microscope and images were captured. 
Assay of MDA content, superoxide dismutase (SOD) and catalase activities

The rats were anaesthetized and then the hearts were harvested (6 animals in sham and alprostadil ( $8 \mu \mathrm{g} / \mathrm{kg}$ ) groups, 5 animals in I/R and alprostadil ( $4 \mu \mathrm{g} / \mathrm{kg}$ ) groups). Supernatants of the left ventricles were prepared as follows. A total of $500 \mathrm{mg}$ tissue from the left ventricle was homogenized with nine volume of ice-cold saline. Subsequently, tissues were centrifuged at $4^{\circ} \mathrm{C}$ for $15 \mathrm{~min}$ at $1,000 \mathrm{~g}$. Supernatants were collected and stored at $-80^{\circ} \mathrm{C}$ for further analysis. The level of protein in the supernatant was measured as previously described ${ }^{14}$ and MDA content in the left ventricles was measured following a previous method ${ }^{15}$. SOD activity was determined using a nitroblue tetrazolium reduction method ${ }^{16}$. Based on $\mathrm{H}_{2} \mathrm{O}_{2}$ decomposition, catalase activity was assayed as previous description ${ }^{17}$.

\section{Western blot}

The rats were anaesthetized and then three hearts in each group were collected. The same location of the left ventricles was harvested. Protein extraction buffer was from Beyotime Institute of Biotechnology (Shanghai, China). The total protein was estimated by Lowry's method. Proteins from the left ventricles $(50 \mu \mathrm{g})$ were separated by SDSPAGE and transferred onto the polyvinylidene difluoride membranes using an electrophoretic transfer system. Membranes were blocked at room temperature for $2 \mathrm{~h}$ with $5 \%$ bovine serum albumin (Sigma-Aldrich; Merck KGaA, Darmstadt, Germany) and subsequently incubated for $24 \mathrm{~h}$ at $4^{\circ} \mathrm{C}$ with antibodies against the following: eNOS (1:500; No. ab76198; Abcam, Cambridge, UK), p-eNOS (Ser1177; 1:1,000; No. ab184154; Abcam, Cambridge, UK). Membranes were subsequently incubated with horseradish peroxidase-conjugated goat anti-rabbit secondary antibody (1:3,000; A0216) at room temperature for $2 \mathrm{~h}$. According to the manufacturer's protocol, enhanced chemiluminescence detection reagents (Beyotime Institute of Biotechnology, Shanghai, China) were used to visualize the bands. Quantitative densitometry was performed with using Gel-Pro Analyzer Version 3.0 (Media Cybernetics company, Maryland, USA).

NO assay

NO levels in the left ventricles were determined using Greiss reagent. Briefly, $100 \mu \mathrm{l}$ supernatant was mixed with an equal volume of Griess reagent (1\% sulfanilamide and $0.1 \%$ naphthylenediamine in $5 \%$ phosphoric acid; Nanching Jiancheng Bioengineering Institute, Nanjing, China). Absorbance was recorded at $540 \mathrm{~nm}$ using an ELISA plate reader (BioTek Instruments, Inc., Winooski, VT, USA). Concentrations of nitrite were calculated in accordance with the standard curve obtained from sodium nitrite.

\section{Statistical analysis}

Data are expressed as the mean \pm standard deviation and statistical significance was determined using one-way analysis of variance followed by least significant difference testing. $P<0.05$ was considered to indicate a statistically significant difference. The SPSS 22.0 Statistical Software (IBM, New York, USA) was used for the analyses.

\section{Results}

Effect of alprostadil on mortality and myocardial infarct size in myocardial $I / R$ rats

The survival rate of rats was $100 \%$ $(18 / 18)$ in the sham group, $85.0 \%(17 / 20)$ in 
the I/R group, $85.0 \%(17 / 20)$ in the alprostadil $(4 \mu \mathrm{g} / \mathrm{kg})$ group and $90.0 \%(18 / 20)$ in the alprostadil $(8 \mu \mathrm{g} / \mathrm{kg})$ group. No significant difference was observed in survival rates among the alprostadil treatment groups and the $I / R$ group. Myocardial $I / R$ induced an infarct in the rat heart (Figure 1). However, treatment with alprostadil at doses of 4 and $8 \mu \mathrm{g} / \mathrm{kg}$ significantly reduced the myocardial infarct size $(P<0.05$; Figure 1$)$.
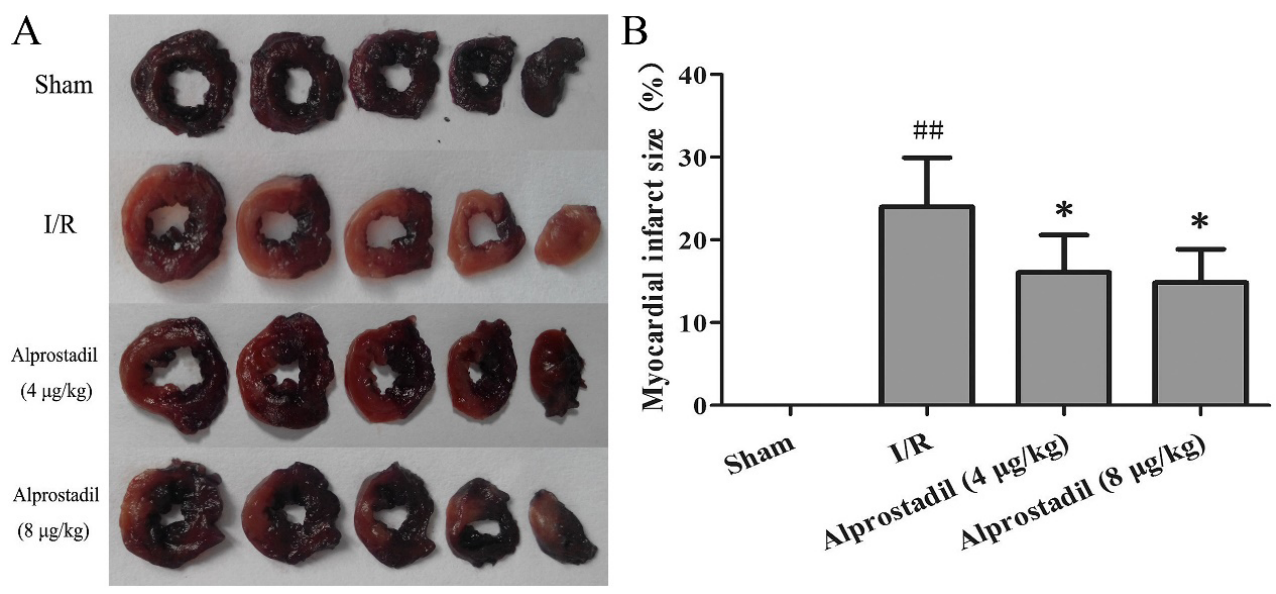

Figure 1 - Effect of alprostadil on myocardial infarct size in I/R rats $(n=6)$. (A) Sections of myocardial tissue stained with triphenyltetrazolium chloride solution. Magnification, x1. (B) Quantified data for myocardial infarct size. ${ }^{\# P} \mathrm{P}<0.01$ vs. sham group; ${ }^{*} \mathrm{P}<0.05$ vs. I/R group. I/R, myocardial ischemia/reperfusion.

\section{Effect of alprostadil on troponin T level in myocardial I/R rats}

To further investigate whether alprostadil attenuates myocardial I/R injury, troponin $T$ levels were measured at the end of reperfusion period. Compared with the sham group, serum troponin $T$ levels in the $I / R$ group were significantly increased $(P<0.01)$. However, rats treated with alprostadil at doses of 4 or $8 \mu \mathrm{g} / \mathrm{kg}$ had significantly lower troponin T levels compared with the $I / R$ group $(P<0.05$ and $P<0.01$, respectively; Figure 2 ).

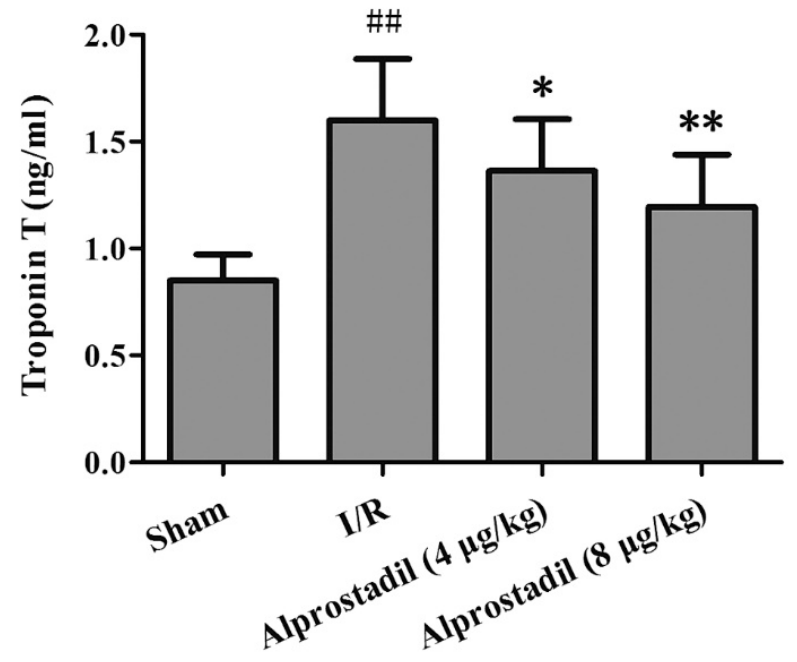

Figure 2 - Effect of alprostadil on serum troponin $T$ in $I / R$ rats $(n=17$ or 18$) .{ }^{\#} P<0.01$ vs. sham group; ${ }^{*} \mathrm{P}<0.05$ and ${ }^{* *} \mathrm{P}<0.01$ vs. I/R group. $\mathrm{I} / \mathrm{R}$, myocardial ischemia/reperfusion. 
Effect of alprostadil on CK-MB and $L D H$ activities in myocardial I/R rats

Serum CK-MB and LDH activities were also assessed to evaluate myocardial damage. The results demonstrated that myocardial I/R resulted in a significant increase in CK-MB and
LDH activities compared with the sham group $(P<0.01)$. However, treatment with $4 \mu \mathrm{g} / \mathrm{kg}$ alprostadil significantly inhibited the increase in LDH activity $(\mathrm{P}<0.05)$ and $8 \mu \mathrm{g} / \mathrm{kg}$ alprostadil significantly inhibited CK-MB and LDH activity in the rat serum $(P<0.05$; Figure 3$)$.
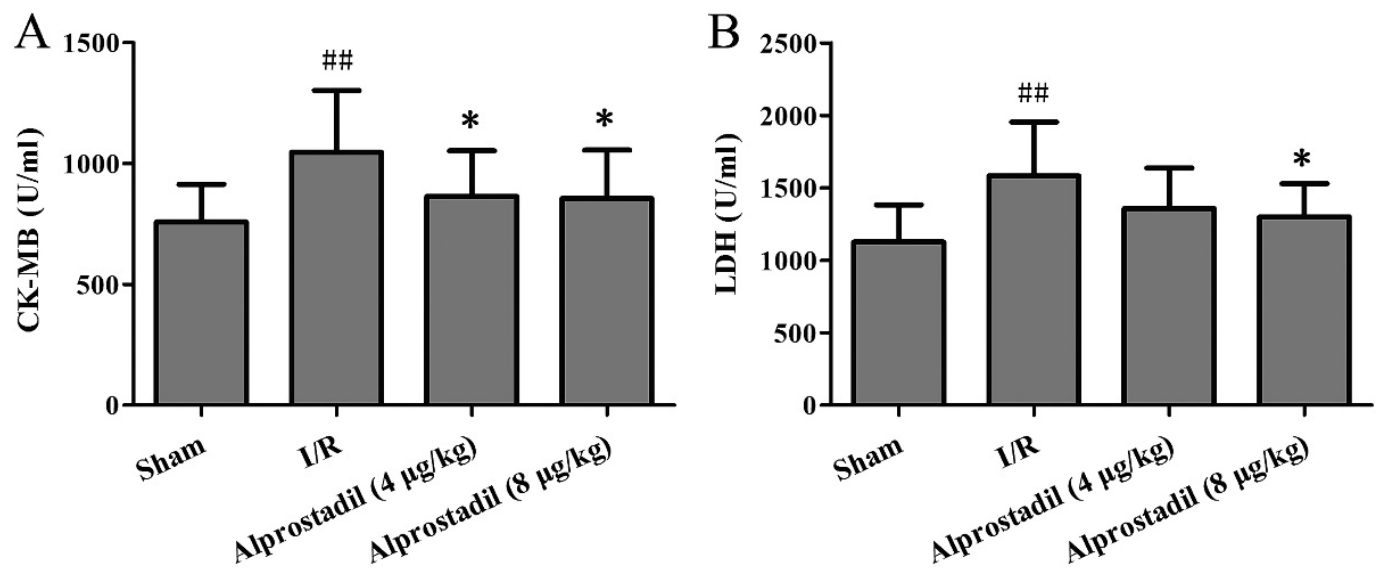

Figure 3 - Effect of alprostadil on serum (A) CK-MB and (B) LDH activity in I/R rats ( $n=17$ or 18$)$. ${ }^{\# \#<0.01 ~ v s . ~}$ sham group; ${ }^{P}<0.05$ vs. I/R group. I/R, myocardial ischemia/reperfusion; CK-MB, creatine kinase-MB; LDH, lactate dehydrogenase.

\section{Effect of alprostadil on histopathological changes in the left ventricles}

Hematoxylin and eosin staining was used to assess histopathological changes in the ischemic heart tissue following reperfusion. Myocardia from the sham group had a normal tissue structure and shape (Figure 4A). Necrosis, infiltration of neutrophil granulocytes, and myocardial and interstitial edema was observed in tissues from the I/R group (Figure $4 B$ ). Rats in the alprostadil groups exhibited mild necrosis and myocardial interstitial edema, accompanied by markedly less infiltration of inflammatory cells compared with the I/R group (Figure $4 C$ and $D$ ).

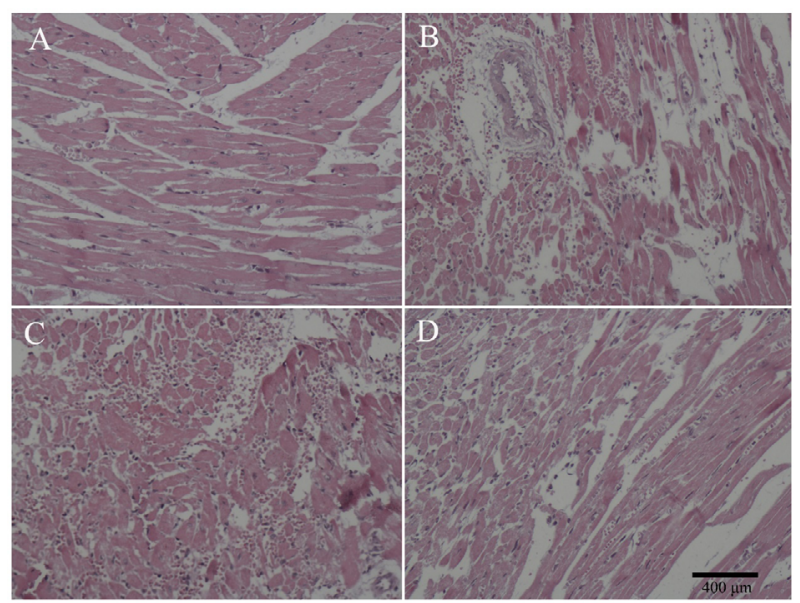

Figure 4 - Effect of alprostadil on histopathological changes in the left ventricles $(n=3)$. Hematoxylin and eosin staining of (A) sham, (B) myocardial ischemia/reperfusion, (C) $4 \mu \mathrm{g} / \mathrm{kg}$ alprostadil and (D) $8 \mu \mathrm{g} / \mathrm{kg}$ alprostadil groups. Bar=400 $\mu \mathrm{m}$. 
Effects of alprostadil on MDA content, SOD and catalase cctivities in the left ventricles

To examine the effect of alprostadil on the myocardial oxidative stress, MDA content in ischemia heart tissue was measured. In the I/R group, MDA content was significantly increased compared with the sham group $(P<0.01$; Figure 5); however, SOD and catalase activities were significantly decreased $(P<0.01$; Figure 6). Treatment with 4 or $8 \mu \mathrm{g} / \mathrm{kg}$ alprostadil significantly reduced the $1 / R$-induced increase in MDA content $(P<0.05$; Figure 5$)$. Treatment with 4 or $8 \mu \mathrm{g} / \mathrm{kg}$ alprostadil significantly increased SOD activity compared with the $\mathrm{I} / \mathrm{R}$ group $(\mathrm{P}<0.05$; Figure $6 \mathrm{~A})$ and $8 \mu \mathrm{g} / \mathrm{kg}$ alprostadil significantly ameliorated the $1 / R-$ induced decrease in catalase activity $(P<0.05$; Figure 6B).

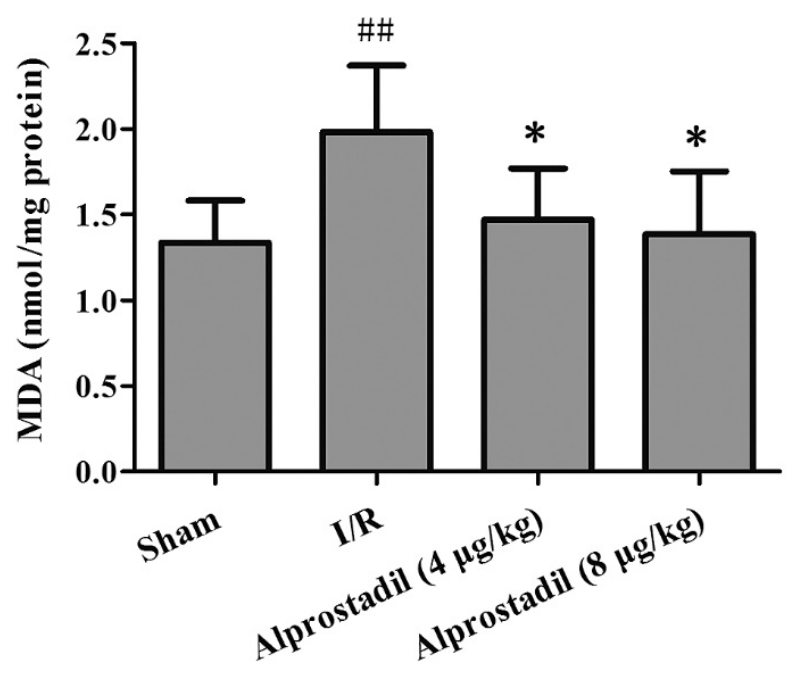

Figure 5 - Effect of alprostadil on MDA content in the left ventricles ( $\mathrm{n}=5$ or 6$)$. ${ }^{\#} \mathrm{P}<0.01$ vs. sham group; ${ }^{*} \mathrm{P}<0.05$ vs. I/R group. $\mathrm{I} / \mathrm{R}$, myocardial ischemia/ reperfusion; MDA, malondialdehyde.
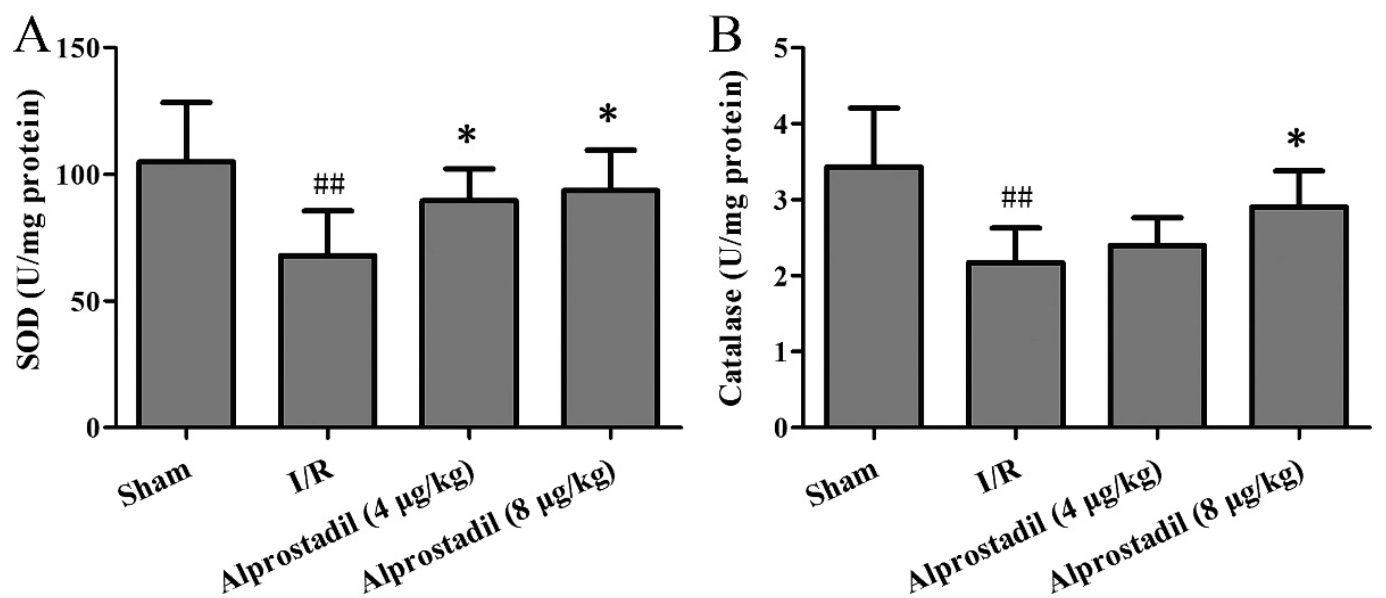

Figure 6 - Effect of alprostadil on (A) SOD and (B) catalase activities in the left ventricles ( $n=5$ or 6$) .{ }^{\prime \prime} P<0.01$ vs. sham group; ${ }^{*} \mathrm{P}<0.05$ vs. I/R group. I/R, myocardial ischemia/reperfusion; SOD, superoxide dismutase.

Effect of alprostadil on the expression of $e$ $p$-eNOS/NOS in the left ventricles

No significant difference was observed in eNOS expression among the groups.
However, p-eNOS levels in the I/R group were significantly decreased compared with the sham group $(P<0.01)$. This decrease was significantly reversed following 4 or $8 \mu \mathrm{g} / \mathrm{kg}$ alprostadil treatment $(P<0.05$; Figure 7$)$. 


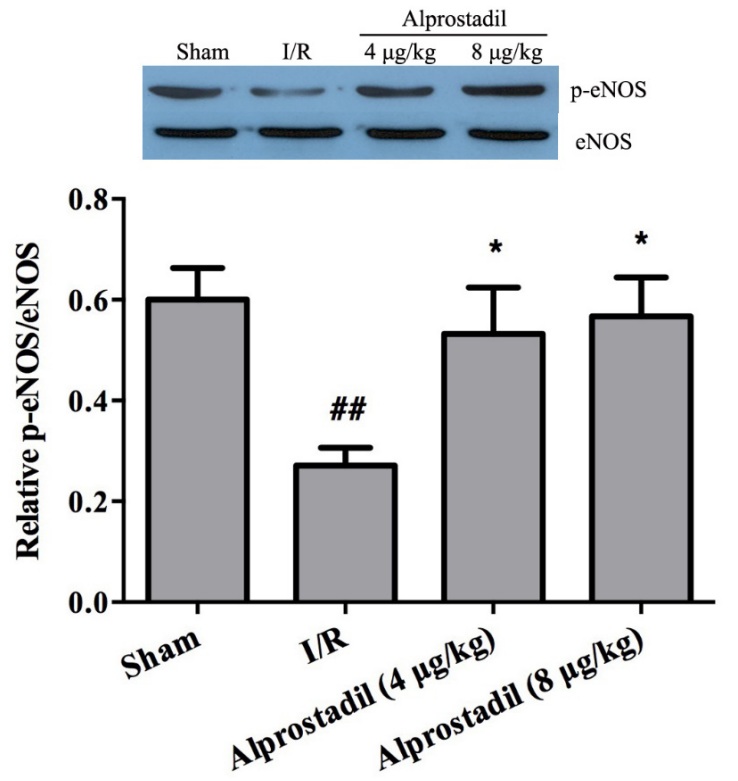

Figure 7 - Effect of alprostadil on p-eNOS/eNOS in the left ventricles $(n=3)$. ${ }^{\#} P<0.01$ vs. sham group; ${ }^{*} \mathrm{P}<0.05$ vs. I/R group. I/R, myocardial ischemia/ reperfusion; eNOS, endothelial nitric oxide synthase 3 .

\section{Effect of alprostadil on NO levels in the left} ventricles

To further investigate the biochemical events associated with alprostadil treatment in the left ventricles, NO production in the ischemic heart was evaluated. NO levels were significantly lower in the I/R group compared with the sham group $(P<0.05)$. However, treatment with 4 or $8 \mu \mathrm{g} / \mathrm{kg}$ alprostadil induced a significant increase in NO levels in the left ventricles compared with the $1 / R$ group $(P<$ 0.01; Figure 8).

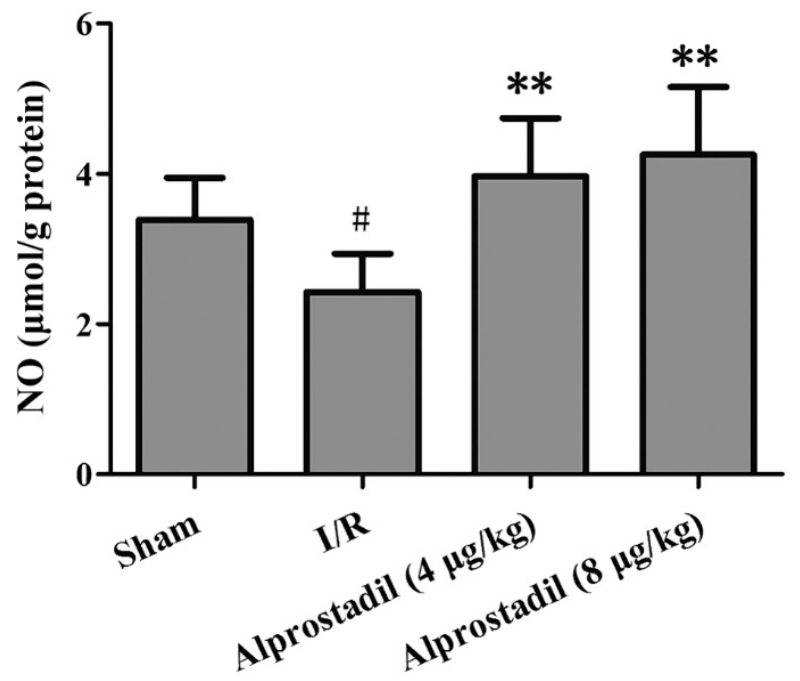

Figure 8 - Effect of alprostadil on NO levels in the left ventricles ( $n=5$ or 6$)$. ${ }^{~} P<0.05$ vs. sham group; ${ }^{* *} \mathrm{P}<0.01$ vs. I/R group. I/R, myocardial ischemia/ reperfusion; NO, nitric oxide.

\section{Discussion}

Inthe presentstudy, itwas demonstrated that treatment with alprostadil reduces myocardial infarct size in a rat myocardial I/R model in vivo. The cardioprotective effect of alprostadil was associated with an increase in SOD and catalase activity and a reduction in MDA content. Furthermore, alprostadil increased the phosphorylation of eNOS and augmented the production of NO. These results suggest that alprostadil may protect cardiomyocytes from myocardial I/R injury, at least in part via the promotion of antioxidant activity and activation of eNOS.

Levels of troponin T, CK-MB and LDH in the serum may be used to determine the degree of injury to cardiomyocytes ${ }^{18}$. The results of the present study revealed that myocardial $I / R$ causes significant elevations in serum levels of troponin T, CK-MB and LDH. However, treatment with alprostadil at doses 
of 4 or $8 \mu \mathrm{g} / \mathrm{kg}$ significantly decreased the myocardial I/R-induced elevations of troponin $\mathrm{T}$ and $\mathrm{CK}-\mathrm{MB}$ and treatment with $4 \mu \mathrm{g} / \mathrm{kg}$ alpostadil significantly reversed myocardial I/R-induced elevations of LDH. Furthermore, the left ventricles of myocardial I/R rats exhibited widespread myocardial structure disorders, including necrosis, infiltration of neutrophil granulocytes, and myocardial and interstitial edema. Treatment with alprostadil alleviated these histopathological changes in the left ventricles. In agreement with our data, a previous study indicates that $\mathrm{PGE}_{1}$ reduces myocardial reperfusion injury in patients with cardiac surgery. Administration of $\mathrm{PGE}_{1}$ inhibits the increases of troponin $\mathrm{T}$ and $\mathrm{CK}-\mathrm{MB}^{19}$. Furthermore, recent study demonstrates that intravenous $\mathrm{PGE}_{1}$ can reduce the incidence of periprocedural myocardial injury in patients with unstable angina undergoing an elective percutaneous coronary ${ }^{20}$. Together, these evidences strongly suggest that alprostadil has cardioprotective properties.

Restored oxygen may lead to the generation of oxygen metabolites, in particular reactive oxygen species, which are key molecules in myocardial I/R injury ${ }^{21}$. SOD and catalase are antioxidant enzymes regulating redox homeostasis. A previous study indicated that treatment with antioxidants, including SOD, catalase and an antioxidation inducer, resulted in reduced myocardial $\mathrm{I} / \mathrm{R}$ damage $^{22}$. In the present study, MDA content was significantly higher and SOD and catalase activities were significantly lower in the myocardial I/R group compared with the sham group. Treatment with alprostadil decreased MDA content, as well as augmented SOD and catalase activity. In line with these results, Hecker and colleagues demonstrate that $P \mathrm{PE}_{1}$ inhibits the neutrophils chemotaxis and oxygen radical release ${ }^{23}$. Together these findings suggest that the cardioprotective property of alprostadil may be associated with its antioxidant activity.
eNOS is expressed in coronary microvessels and cardiomyocytes and produces low concentrations of cytoprotective NO in normal hearts. Phosphorylation and dephosphorylation are major posttranslational regulators that influence eNOS activity. Phosphorylation of eNOS at Ser1177 increases its activity, thus p-eNOS increases the availability of $\mathrm{NO}^{24}$. NO serves a key role in the alleviation of myocardial I/R injury, preventing the vasospasm of coronary arteries and inhibiting the aggregation of platelets. In the present study, western blotting was used to determine the effect of alprostadil on eNOS phosphorylation. The results demonstrated that eNOS phosphorylation was significantly increased in the groups treated with alprostadil. Alprostadil was also enhanced NO production in the left ventricles of myocardial I/R rats. Therefore, another mechanism by which alprostadil ameliorates myocardial I/R injury may be associated with increased NO following increased eNOS phosphorylation.

When interpreting the results of the present study, its potential limitations must be considered. Firstly, the present study was performed using an animal model. Further investigations are required to assess the potential of alprostadil for the treatment of humans with myocardial infarction. Secondly, protein kinase B (Akt) may mediate the phosphorylation of eNOS ${ }^{25}$. Although the downstream target of Akt, eNOS, was examined in the present study, the effect of alprostadil on the expression of Akt requires further investigation.

\section{- Conclusions}

The alprostadil attenuates myocardial $I / R$ injury in rats. The protective effects of alprostadil are achieved at least in part by promoting antioxidant activity and eNOS activation. 


\section{References}

1. Simon AS, Vijayakumar T. Molecular studies on coronary artery disease-a review. Indian J Clin Biochem. 2013;28:215-26. PMID: 24426215.

2. Yellon DM, Hausenloy DJ. Reducing myocardial infarct size: challenges and future opportunities. Bulluck $\mathrm{H}$ Heart. 2016;102(5):341-8. PMID: 26674987.

3. Canty JM Jr, Suzuki G. Myocardial perfusion and contraction in acute ischemia and chronic ischemic heart disease. J Mol Cell Cardiol. 2012;52(4):822-31. PMID: 21889943.

4. Jones SP, Greer JJ, Kakkar AK, Ware PD, Turnage $\mathrm{RH}$, Hicks $\mathrm{M}$, van Haperen $\mathrm{R}$, de Crom R, Kawashima S, Yokoyama M, Lefer DJ. Endothelial nitric oxide synthase overexpression attenuates myocardial reperfusion injury. Am J Physiol Heart Circ Physiol. 2004;286:276-82. PMID: 12969888.

5. Bice JS, Jones BR, Chamberlain GR, Baxter GF. Nitric oxide treatments as adjuncts to reperfusion in acute myocardial infarction: a systematic review of experimental and clinical studies. Basic Res Cardiol. 2016;111(2):23. PMID: 26912064.

6. Talukder MA, Yang F, Shimokawa H, Zweier $\mathrm{JL}$. eNOS is required for acute in vivo ischemic preconditioning of the heart: effects of ischemic duration and sex. Am J Physiol Heart Circ Physiol. 2010;299:437-45. PMID: 20525875.

7. Gezginci-Oktayoglu S, Orhan N, Bolkent S. Prostaglandin-E1 has a protective effect on renal ischemia/reperfusioninduced oxidative stress and inflammation mediated gastric damage in rats. Int Immunopharmacol. 2016;36:142-50. PMID: 27135545.

8. Acciavatti A, Laghi Pasini F, Capecchi PL, Messa GL, Lazzerini PE, De Giorgi L, Acampa $M$, Di Perri T. Effects of alprostadil on blood rheology and nucleoside metabolism in patients affected with lower limb chronic ischaemia. Clin Hemorheol Microcirc. 2001;24:49-57. PMID: 11345234.

9. Luo C, Li T, Zhang C, Chen Q, Li Z, Liu J, Wang $Y$. Therapeutic effect of alprostadil in diabetic nephropathy: possible roles of angiopoietin-2 and IL-18. Cell Physiol Biochem. 2014;34(3):916-28. PMID:

\section{3.}

10.Soares BL, Freitas MA, Montero EF, Pitta GB, Miranda F, Jr. Alprostadil attenuates inflammatory aspects and leucocytes adhesion on renal ischemia and reperfusion injury in rats. Acta Cir Bras. 2014;2:55-60. PMID: 25229516.

11. Hsieh CC, Hsieh SC, Chiu JH, Wu YL. Protective effects of $\mathrm{N}$-acetylcysteine and a prostaglandin E1 analog, alprostadil, against hepatic ischemia: reperfusion injury in rats. J Tradit Complement Med. 2014;4:64-71. PMID: 24872935.

12. Milcan A, Arslan E, Bagdatoglu OT, Bagdatoglu C, Polat G, Kanik A, Talas DU, Kuyurtar $F$. The effect of alprostadil on ischemia-reperfusion injury of peripheral nerve in rats. Pharmacol Res. 2004;49:6772. PMID: 14597154.

13.Xia R, Zhao B, Wu Y, Hou JB, Zhang L, Xu JJ, Xia ZY. Ginsenoside Rb1 preconditioning enhances eNOS expression and attenuates myocardial ischemia/reperfusion injury in diabetic rats. J Biomed Biotechnol. 2011;767930. PMID: 22013385.

14.Lowry OH, Rosebrough NJ, Farr AL, Randall RJ. Protein measurement with the Folin phenol reagent. J Biol Chem. 1951;193:26575. PMID: 14907713.

15. Ohkawa H, Ohishi N, Yagi K. Assay for lipid peroxides in animal tissues by thiobarbituric acid reaction. Anal Biochem. 1979;95:3518. PMID: 36810.

16.McCord JM, Fridovich I. Superoxide dismutase. An enzymic function for erythrocuprein (hemocuprein). J Biol Chem. 1969;244:6049-55. PMID: 5389100.

17.Aebi H. Catalase in vitro. Methods Enzymol. 1984;105:121-6. PMID: 6727660.

18.Dong W, Yang R, Yang J, Yang J, Ding J, Wu H, Zhang J. Resveratrol pretreatment protects rat hearts from ischemia/reperfusion injury partly via a NALP3 inflammasome pathway. Int J Clin Exp Pathol. 2015;8:8731-41. PMID: 26464617.

19. Kawamura T, Nara N, Kadosaki M, Inada $\mathrm{K}$, Endo S. Prostaglandin E1 reduces myocardial reperfusion injury by inhibiting proinflammatory cytokines production during cardiac surgery. Crit Care Med. 2000;28(7):2201-8. PMID: 10921541.

20.Fan Y, Jiang Y, Fu X, Cai J, Wang Y, Li W, Gu $X$, Xing K, Bai S, Bi X. Effects of liposomal 
prostaglandin E1 on periprocedural myocardial injury in patients with unstable angina undergoing an elective percutaneous coronary intervention. Coron Artery Dis. 2015;26(8):671-7. PMID: 26267747.

21.Yu J, Wang L, Akinyi M, Li Y, Duan Z, Zhu Y, Fan G. Danshensu protects isolated heart against ischemia reperfusion injury through activation of Akt/ERK1/2/Nrf2 signaling. Int J Clin Exp Med. 2015;8:14793-804. PMID: 26628961.

22.Piao CS, Gao S, Lee GH, Kim do S, Park BH, Chae SW, Chae HJ, Kim SH. Sulforaphane protects ischemic injury of hearts through antioxidant pathway and mitochondrial K(ATP) channels. Pharmacol Res. 2010;61:342-8. PMID: 19948220.

23. Hecker G1, Ney P, Schrör K. Cytotoxic enzyme release and oxygen centered radical formation in human neutrophils are selectively inhibited by E-type prostaglandins but not by PGI2. Naunyn Schmiedebergs Arch Pharmacol. 1990;341(4):308-15. PMID: 2159112.

24.Xia T, Guan W, Fu J, Zou X, Han Y, Chen C, Zhou L, Zeng $C$, Wang WE. Tirofiban induces vasorelaxation of the coronary artery via an endothelium-dependent NO-cGMP signaling by activating the PI3K/Akt/eNOS pathway. Biochem Biophys Res Commun. 2016;474(3):599-605. PMID: 27018249.

25.Zancheta D, Troiano JA, Potje SR, Cavalari P, Sumida DH, Antoniali C. The PI3K-Akt-eNOS pathway is involved in aortic hyporeactivity to Phenylephrine associated with late pregnancy in spontaneously hypertensive rats. Life Sci. 2015;122:78-86. PMID: 25534440.

\section{Correspondence:}

Dayuan Sui

College of Pharmacy, Department of Cardiovascular Medicine

First Hospital Jilin University

Changchun, Jilin 130021, P.R. China

suidy@jlu.edu.cn

Received: Aug 16, 2018

Review: Oct 19, 2018

Accepted: Nov 17, 2018
Conflict of interest: none

Financial source: Industrial Technology Research Project of Jilin Province (grant no. 3J113AX13432)
${ }^{1}$ Research performed at Pharmacology, College of Pharmacy, Jilin University, Changchun, Jilin, P.R., China. 